\title{
THE DISCRETE COAGULATION EQUATIONS WITH MULTIPLE FRAGMENTATION
}

\author{
PHILIPPE LAURENÇOT \\ CNRS et Institut Elie Cartan, Nancy, Université de Nancy I, BP 239, \\ F-54506 Vandouvre-lès-Nancy cedex, France and \\ Mathématiques pour l'Industrie et la Physique, Université Paul Sabatier-Toulouse 3, \\ 118 route de Narbonne, F-31062 Toulouse cedex 4, \\ France (laurencot@mip.ups-tlse.fr)
}

(Received 16 March 2000)

\begin{abstract}
We present an alternative proof of the existence of density-conserving solutions to the discrete coagulation-fragmentation equations when the coagulation rates grow at most linearly. The proof relies on the study of the propagation of some moments of the solutions to approximating equations and simplifies the previous argument of Ball and Carr which involves rather delicate estimates. The case of multiple fragmentation is also considered, and the question of uniqueness as well.
\end{abstract}

Keywords: coagulation; multiple fragmentation; existence; propagation of moments

AMS 2000 Mathematics subject classification: Primary 34A34; 82C22

\section{Introduction}

The coagulation-fragmentation equations are a model for the dynamics of cluster growth and describe the time evolution of a system of clusters undergoing coalescence and breakup simultaneously. In the model considered in this paper the clusters are assumed to be discrete, that is, they consist of a finite number of elementary particles. The basic reactions between clusters that are taken into account are the coalescence of two clusters to form a larger one and the breakage of a cluster into smaller pieces. Other effects (multiple coagulation, spatial fluctuations, etc.) are neglected. Denoting by $c_{i}(t), i \in \mathbb{N} \backslash\{0\}$, the number of clusters made of $i$ particles ( $i$-clusters) per unit volume at time $t \geqslant 0$, the discrete coagulation-fragmentation equations read

$$
\begin{gathered}
\frac{\mathrm{d} c_{i}}{\mathrm{~d} t}=\frac{1}{2} \sum_{j=1}^{i-1}\left(\phi_{j, i-j} c_{j} c_{i-j}-\psi_{j, i-j} c_{i}\right)-\sum_{j=1}^{\infty}\left(\phi_{i, j} c_{i} c_{j}-\psi_{i, j} c_{i+j}\right), \\
c_{i}(0)=c_{i}^{0},
\end{gathered}
$$

for $i \geqslant 1$, under the additional assumption that only binary fragmentation is allowed for. Here the coagulation rates $\phi_{i, j}$ and the fragmentation rates $\psi_{i, j}$ are non-negative real 
numbers satisfying

$$
\phi_{i, j}=\phi_{j, i} \quad \text { and } \quad \psi_{i, j}=\psi_{j, i}, \quad i, j \geqslant 1 .
$$

On the right-hand side of (1.1) the first term accounts for the formation of $i$-clusters by binary coalescence of smaller ones and the second one for the fragmentation of $i$-clusters into two smaller ones. The third term describes the depletion of $i$-clusters by coagulation with other clusters, while the fourth term represents the creation of $i$-clusters resulting from the breakage of larger ones. System (1.1) without fragmentation $\left(\psi_{i, j}=0\right)$ was originally introduced by Smoluchowski $[\mathbf{2 1}, \mathbf{2 2}]$ and we refer to $[\mathbf{6}]$ for a derivation of (1.1) and some physical background. Observe that, since particles are neither created nor destroyed in the reactions described by (1.1), the density

$$
\varrho(t)=\sum_{i=1}^{\infty} i c_{i}(t)
$$

is expected to be conserved through time evolution. It is, however, well known by now that, in the absence of fragmentation, there are physically relevant coagulation rates for which density conservation breaks down in finite time, a phenomenon known as gelation $[\mathbf{8}, \mathbf{9}, \mathbf{1 2}, \mathbf{1 5}, \mathbf{1 6}]$. We will, however, not consider this issue here and concentrate on the case when the coagulation rates satisfy

$$
\phi_{i, j} \leqslant K(i+j), \quad i, j \geqslant 1 .
$$

Under assumption (1.5) on the coagulation rates and without any further assumption on the fragmentation rates, existence of a solution to (1.1), (1.2) satisfying $\varrho(t)=\varrho(0)$ for $t \geqslant 0$ has been shown by Ball and Carr for any initial datum $c^{0}=\left(c_{i}^{0}\right)$ in $X^{+}[\mathbf{1}]$. Here, $X^{+}$denotes the positive cone of the Banach space $X$ defined by

$$
X=\left\{x=\left(x_{i}\right)_{i \geqslant 1} \in \mathbb{R}^{\mathbb{N} \backslash\{0\}}, \sum_{i=1}^{\infty} i\left|x_{i}\right|<\infty\right\},
$$

with the norm

$$
\|x\|_{X}=\sum_{i=1}^{\infty} i\left|x_{i}\right|
$$

that is,

$$
X^{+}=\left\{x \in X, x_{i} \geqslant 0 \text { for each } i \geqslant 1\right\} .
$$

The existence proof in [1] is carried out by taking a limit of solutions to approximating finite-dimensional systems of ordinary differential equations and requires delicate estimates in order to recover the infinite series on the right-hand side of (1.1) (see [1, pp. 211-215]).

Our aim in this paper is to present a simpler proof of [1, Theorems 2.4 and 2.5]. Our approach relies on the study of the propagation of the moments $\sum g_{i} c_{i}$ for approximating solutions. Under assumption (1.5) the propagation of moments for approximating solutions has been investigated in $[\mathbf{7}, \mathbf{2 4}]$ in the absence of fragmentation and in $[\mathbf{2}-\mathbf{4}]$ for the 
full model (1.1) with $g_{i}=i^{m}, m \geqslant 1$, and we will generalize these results to a wider class of sequences $\left(g_{i}\right)$. Combining such estimates with a refined version of the de la ValléePoussin theorem $[\mathbf{5}, \mathbf{1 4}]$ then yields an alternative proof of [1, Theorems 2.4 and 2.5]. Our method is actually sufficiently flexible that we can also handle multiple fragmentation [17] and obtain a new existence result for this model. Let us finally mention that several existence results have been obtained for (1.1), (1.2) when the coagulation rates do not satisfy (1.5) but the weaker condition

$$
\phi_{i, j} \leqslant K i j, \quad i, j \geqslant 1
$$

either in the absence of fragmentation $[\mathbf{1 2}, \mathbf{1 5}, \mathbf{1 8}, \mathbf{1 9}, \mathbf{2 4}]$ or for some classes of fragmentation rates $[\mathbf{4}, \mathbf{2 3}]$.

We now describe the contents of this paper: $\S 2$ introduces the discrete coagulation equations with multiple fragmentation together with the statement of our main results. The finite-dimensional systems of ordinary differential equations approximating (1.1) are described in $\S 3$ and the propagation of the moments of their solutions is investigated. Section 4 is devoted to the proof of our existence result and a partial uniqueness result is given in $\S 5$.

\section{Main results}

As already mentioned, we consider a generalized version of (1.1) which allows multiple fragmentation $[\mathbf{1 7}]$ and reads

$$
\begin{gathered}
\frac{\mathrm{d} c_{i}}{\mathrm{~d} t}=\frac{1}{2} \sum_{j=1}^{i-1} \phi_{j, i-j} c_{j} c_{i-j}-a_{i} c_{i}-\sum_{j=1}^{\infty}\left(\phi_{i, j} c_{i} c_{j}-a_{i+j} b_{i+j, i} c_{i+j}\right), \\
c_{i}(0)=c_{i}^{0},
\end{gathered}
$$

for $i \geqslant 1$. Here $\phi_{i, j}$ still denotes the coagulation rates, while $a_{i}$ gives the rate of fragmentation of $i$-clusters (with $a_{1}=0$ ) and $b_{i, j}$ is the average number of particles of size $j$ produced upon the breakup of an $i$-cluster. Conservation of mass during the fragmentation reaction implies

$$
\sum_{j=1}^{i-1} j b_{i, j}=i, \quad i \geqslant 2,
$$

and such a model clearly allows the breakage of $i$-clusters into more than two pieces.

Throughout the paper the assumptions made on the data $\left(\phi_{i, j}\right),\left(a_{i}\right)$ and $\left(b_{i, j}\right)$ are the following: there is a positive real number $K$ such that

$$
\begin{gathered}
0 \leqslant \phi_{i, j}=\phi_{j, i} \leqslant K(i+j), \quad i, j \geqslant 1, \\
a_{1}=0 \quad \text { and } \quad a_{i} \geqslant 0, \quad i \geqslant 2, \\
\left(b_{i, j}\right) \in[0,+\infty)^{i-1} \text { and } \sum_{j=1}^{i-1} j b_{i, j}=i, \quad i \geqslant 2 .
\end{gathered}
$$


Remark 2.1. The discrete coagulation-fragmentation equations (1.1) are actually a particular case of (2.1). Indeed, given fragmentation rates $\left(\psi_{i, j}\right)$ satisfying (1.3), we recover (1.1) from (2.1) by setting

$$
a_{i}=\frac{1}{2} \sum_{j=1}^{i-1} \psi_{j, i-j}, \quad i \geqslant 1,
$$

with

$$
b_{i, j}=\frac{\psi_{j, i-j}}{a_{i}} \quad \text { if } a_{i} \neq 0 .
$$

We also point out that, in that case, the symmetry of $\left(\psi_{i, j}\right)$ entails that $b_{i, j}=b_{i, i-j}$, $1 \leqslant j \leqslant i-1$.

Before stating our existence result we specify what we mean by a solution to (2.1), $(2.2)$.

Definition 2.2. Let $T \in(0,+\infty]$ and $c^{0}=\left(c_{i}^{0}\right)_{i \geqslant 1}$ be a sequence of non-negative real numbers. A solution $c=\left(c_{i}\right)_{i \geqslant 1}$ to $(2.1),(2.2)$ on $[0, T)$ is a sequence of non-negative continuous functions satisfying, for each $i \geqslant 1$ and $t \in(0, T)$,

(i) $c_{i} \in \mathcal{C}([0, T)), \sum_{j=1}^{\infty} \phi_{i, j} c_{j} \in L^{1}(0, t)$ and $\sum_{j=i+1}^{\infty} a_{j} b_{j, i} c_{j} \in L^{1}(0, t)$,

(ii) and there holds

$$
\begin{aligned}
c_{i}(t)=c_{i}^{0}+\int_{0}^{t}\left(\frac{1}{2} \sum_{j=1}^{i-1} \phi_{j, i-j} c_{j}(s)\right. & c_{i-j}(s)-a_{i} c_{i}(s) \\
& \left.-\sum_{j=1}^{\infty}\left(\phi_{i, j} c_{i}(s) c_{j}(s)-a_{i+j} b_{i+j, i} c_{i+j}(s)\right)\right) \mathrm{d} s .
\end{aligned}
$$

Our existence result then reads as follows.

Theorem 2.3. Consider $c^{0} \in X^{+}$(where $X^{+}$is defined in (1.6)). Under the assumptions (2.3)-(2.5) there is at least one solution $c$ to $(2.1),(2.2)$ on $[0,+\infty)$ satisfying

$$
\|c(t)\|_{X}=\left\|c^{0}\right\|_{X}, \quad t \in[0,+\infty) .
$$

In other words, the density of the solution $c$ is conserved through time evolution.

We actually prove a more precise result. We first introduce some notation. We denote by $\mathcal{K}_{1}$ the set of non-negative and convex functions $U \in \mathcal{C}^{1}([0,+\infty)) \cap W_{\text {loc }}^{2, \infty}(0,+\infty)$ such that $U(0)=0, U^{\prime}(0) \geqslant 0$ and $U^{\prime}$ is a concave function. We next denote by $\mathcal{K}_{1, \infty}$ the set of functions $U \in \mathcal{K}_{1}$ satisfying, in addition,

$$
\lim _{r \rightarrow+\infty} U^{\prime}(r)=\lim _{r \rightarrow+\infty} \frac{U(r)}{r}=+\infty .
$$


We finally introduce the set $\mathcal{K}_{2}$ of non-negative and convex functions $U \in \mathcal{C}^{2}([0,+\infty))$ such that $U(0)=U^{\prime}(0)=0$ and $U^{\prime}$ is a convex function satisfying the $\Delta_{2}$-condition, that is,

$$
U^{\prime}(2 r) \leqslant k_{U} U^{\prime}(r), \quad r \in[0,+\infty),
$$

for some $k_{U}>0$. It follows from [11, Theorem 4.1] that, if $U \in \mathcal{K}_{2}$, there exists $\ell_{U}>0$ such that

$$
r U^{\prime \prime}(r) \leqslant \ell_{U} U^{\prime}(r), \quad r \in[0,+\infty) .
$$

Remark 2.4. It is clear that $r \mapsto r^{m}$ belongs to $\mathcal{K}_{1, \infty}$ if $m \in(1,2]$ and to $\mathcal{K}_{2}$ if $m \geqslant 2$.

Our next result then reads as follows.

Theorem 2.5. Consider $c^{0} \in X^{+}$and assume that there is $U \in \mathcal{K}_{1} \cup \mathcal{K}_{2}$ such that

$$
\sum_{i=1}^{\infty} U(i) c_{i}^{0}<\infty
$$

Under the assumptions (2.3)-(2.5) there is at least one solution $c$ to $(2.1),(2.2)$ on $[0,+\infty)$ satisfying (2.6) and, for each $T \in(0,+\infty)$,

$$
\sup _{t \in[0, T]} \sum_{i=1}^{\infty} U(i) c_{i}(t)<\infty .
$$

Consequently, if the initial datum has some finite moment (in a suitable class), there is at least one solution to (2.1), (2.2) enjoying the same property for all times.

Remark 2.6. When applied to the discrete coagulation-fragmentation equations (1.1), (1.2), Theorem 2.3 is nothing but [1, Theorems 2.4 and 2.5], while Theorem 2.5 extends [3, Theorem 3.3].

From now on we assume that the rate coefficients $\left(\phi_{i, j}\right),\left(a_{i}\right)$ and $\left(b_{i, j}\right)$ are given and satisfy the assumptions (2.3)-(2.5). We also fix $c^{0} \in X^{+}$.

\section{Approximating systems}

As in previous works on similar equations, existence of solutions to (2.1), (2.2) follows by taking a limit of solutions to finite-dimensional systems of ordinary differential equations obtained by truncation of (2.1). More precisely, given $N \geqslant 3$, we consider the following system of $N$ ordinary differential equations:

$$
\begin{gathered}
\frac{\mathrm{d} c_{i}^{N}}{\mathrm{~d} t}=\frac{1}{2} \sum_{j=1}^{i-1} \phi_{j, i-j} c_{j}^{N} c_{i-j}^{N}-a_{i} c_{i}^{N}-\sum_{j=1}^{N-i}\left(\phi_{i, j} c_{i}^{N} c_{j}^{N}-a_{i+j} b_{i+j, i} c_{i+j}^{N}\right), \\
c_{i}^{N}(0)=c_{i}^{0},
\end{gathered}
$$

for $i \in\{1, \ldots, N\}$. Proceeding as in [1, Lemmas 2.1 and 2.2] we obtain the following result. 
Lemma 3.1. For each $N \geqslant 3$ system (3.1), (3.2) has a unique solution

$$
c^{N}=\left(c_{i}^{N}\right)_{1 \leqslant i \leqslant N} \in \mathcal{C}^{1}\left([0,+\infty) ; \mathbb{R}^{N}\right)
$$

with $c_{i}^{N}(t) \geqslant 0$ for $1 \leqslant i \leqslant N$ and $t \geqslant 0$. Furthermore, there holds

$$
\sum_{i=1}^{N} i c_{i}^{N}(t)=\sum_{i=1}^{N} i c_{i}^{0}, \quad t \in[0,+\infty)
$$

and, if $\left(g_{i}\right) \in \mathbb{R}^{N}$,

$\sum_{i=1}^{N} g_{i} \frac{\mathrm{d} c_{i}^{N}}{\mathrm{~d} t}=\frac{1}{2} \sum_{i=1}^{N} \sum_{j=1}^{N-i}\left(g_{i+j}-g_{i}-g_{j}\right) \phi_{i, j} c_{i}^{N} c_{j}^{N}-\sum_{i=2}^{N}\left(g_{i}-\sum_{j=1}^{i-1} g_{j} b_{i, j}\right) a_{i} c_{i}^{N}$.

Our aim is now to estimate the propagation of moments of the solutions $c^{N}$ to (3.1), (3.2). We shall, however, first derive some properties of the sequence $(U(i))_{i \geqslant 1}$ for $U \in$ $\mathcal{K}_{1} \cup \mathcal{K}_{2}$.

Lemma 3.2. Consider $U \in \mathcal{K}_{1} \cup \mathcal{K}_{2}$. There is a constant $m_{U}$ depending only on $U$ such that

$$
(i+j)(U(i+j)-U(i)-U(j)) \leqslant m_{U}(i U(j)+j U(i)), \quad i, j \geqslant 1 .
$$

The inequality (3.5) has already been noted for $U(r)=r^{m}, m \geqslant 1$, in [2, Lemma 2.3] and for $U \in \mathcal{K}_{1}$ in [13]. We recall the proof of the latter below for completeness. Our proof also differs from the one of [2, Lemma 2.3], which uses the homogeneity of the power functions.

Proof. Assume first that $U \in \mathcal{K}_{1}$ and consider $i, j \geqslant 1$. For $\rho \in[0, i]$ and $\sigma \in[0, j]$ the concavity of $U^{\prime}$ yields

$$
\begin{aligned}
& U^{\prime}(\rho+\sigma)-U^{\prime}(\rho) \geqslant \sigma U^{\prime \prime}(\rho+\sigma), \\
& U^{\prime}(\rho+\sigma)-U^{\prime}(\sigma) \geqslant \rho U^{\prime \prime}(\rho+\sigma) .
\end{aligned}
$$

Therefore,

$$
(\rho+\sigma) U^{\prime \prime}(\rho+\sigma)+2 U^{\prime}(\rho+\sigma) \leqslant 4 U^{\prime}(\rho+\sigma)-U^{\prime}(\rho)-U^{\prime}(\sigma) .
$$

Again using the concavity of $U^{\prime}$ we have

$$
U^{\prime \prime}(\tau) \geqslant U^{\prime \prime}(\tau+\sigma), \quad \tau \in(0,+\infty) .
$$

Integrating the above inequality with respect to $\tau$ over $(0, \rho)$ we obtain, since $U^{\prime}(0) \geqslant 0$,

$$
U^{\prime}(\rho+\sigma) \leqslant U^{\prime}(\rho)+U^{\prime}(\sigma) .
$$

Combining (3.6) and (3.7) we conclude that

$$
(\rho+\sigma) U^{\prime \prime}(\rho+\sigma)+2 U^{\prime}(\rho+\sigma) \leqslant 3\left(U^{\prime}(\rho)+U^{\prime}(\sigma)\right) .
$$


Now, as

$$
(i+j) U(i+j)-i U(i)-j U(j)=\int_{0}^{i} \int_{0}^{j}\left((\rho+\sigma) U^{\prime \prime}(\rho+\sigma)+2 U^{\prime}(\rho+\sigma)\right) \mathrm{d} \rho \mathrm{d} \sigma,
$$

we infer from (3.8) that

$$
(i+j) U(i+j)-i U(i)-j U(j) \leqslant 3(i U(j)+j U(i))
$$

from which (3.5) follows (with $m_{U}=2$ ).

We now consider $U \in \mathcal{K}_{2}$ and $i, j \geqslant 1$. For $\rho \in[0, i]$ and $\sigma \in[0, j]$ the convexity of $U^{\prime}$ ensures that

$$
\begin{aligned}
& U^{\prime}(\rho)-U^{\prime}(\rho+\sigma) \geqslant-\sigma U^{\prime \prime}(\rho+\sigma), \\
& U^{\prime}(\sigma)-U^{\prime}(\rho+\sigma) \geqslant-\rho U^{\prime \prime}(\rho+\sigma) .
\end{aligned}
$$

We infer from (2.9) and the above inequalities that

$$
(\rho+\sigma) U^{\prime \prime}(\rho+\sigma)+2 U^{\prime}(\rho+\sigma) \leqslant 2 \ell_{U} U^{\prime}(\rho+\sigma)+U^{\prime}(\rho)+U^{\prime}(\sigma) .
$$

Next we use the convexity of $U^{\prime}$ and (2.8) to obtain

$$
\begin{aligned}
(\rho+\sigma) U^{\prime \prime}(\rho+\sigma)+2 U^{\prime}(\rho+\sigma) & \leqslant U^{\prime}(\rho)+U^{\prime}(\sigma)+\ell_{U}\left(U^{\prime}(2 \rho)+U^{\prime}(2 \sigma)\right) \\
& \leqslant\left(1+k_{U} \ell_{U}\right)\left(U^{\prime}(\rho)+U^{\prime}(\sigma)\right) .
\end{aligned}
$$

We then proceed as above to obtain (3.5) (with $m_{U}=k_{U} \ell_{U}$ ).

We are now in a position to state and prove the main result of this section.

Proposition 3.3. Consider $T \in(0,+\infty)$ and $U \in \mathcal{K}_{1} \cup \mathcal{K}_{2}$. There is a constant $\gamma_{T}$ depending only on $K, U,\left\|c^{0}\right\|_{X}$ and $T$ such that, for each $N \geqslant 3$ and $t \in[0, T]$, there holds

$$
\begin{gathered}
\sum_{i=1}^{N} U(i) c_{i}^{N}(t) \leqslant \gamma_{T} \sum_{i=1}^{N} U(i) c_{i}^{0} \\
0 \leqslant \int_{0}^{T} \sum_{i=1}^{N-1} i \sum_{j=i+1}^{N}\left(\frac{U(j)}{j}-\frac{U(i)}{i}\right) a_{j} b_{j, i} c_{j}^{N}(s) \mathrm{d} s \leqslant \gamma_{T} \sum_{i=1}^{N} U(i) c_{i}^{0} .
\end{gathered}
$$

Proof. For $N \geqslant 3$ and $t \in[0, T]$ we put

$$
M_{U}^{N}(t)=\sum_{i=1}^{N} U(i) c_{i}^{N}(t) .
$$


It follows from (3.4), (2.3), (3.5) and (3.3) that

$$
\begin{gathered}
\frac{\mathrm{d} M_{U}^{N}}{\mathrm{~d} t} \leqslant \frac{1}{2} K \sum_{i=1}^{N} \sum_{j=1}^{N-i}(i+j)(U(i+j)-U(i)-U(j)) c_{i}^{N} c_{j}^{N} \\
-\sum_{i=2}^{N}\left(U(i)-\sum_{j=1}^{i-1} U(j) b_{i, j}\right) a_{i} c_{i}^{N} \\
\leqslant \frac{1}{2} K m_{U} \sum_{i=1}^{N} \sum_{j=1}^{N-i}(i U(j)+j U(i)) c_{i}^{N} c_{j}^{N} \\
-\sum_{i=2}^{N}\left(U(i)-\sum_{j=1}^{i-1} U(j) b_{i, j}\right) a_{i} c_{i}^{N} \\
\frac{\mathrm{d} M_{U}^{N}}{\mathrm{~d} t} \leqslant K m_{U}\left\|c^{0}\right\|_{X} M_{U}^{N}-\sum_{i=2}^{N}\left(U(i)-\sum_{j=1}^{i-1} U(j) b_{i, j}\right) a_{i} c_{i}^{N} .
\end{gathered}
$$

Observe next that, since $U(0)=0$, the convexity of $U$ and (2.5) yield

$$
\sum_{j=1}^{i-1} U(j) b_{i, j} \leqslant U(i)
$$

and the second term on the right-hand side of (3.11) is non-negative. We therefore deduce from (3.11) and the Gronwall lemma that (3.9) holds true. We next combine (3.9), (3.11) and (3.12) to obtain

$$
0 \leqslant \int_{0}^{T} \sum_{i=2}^{N}\left(U(i)-\sum_{j=1}^{i-1} U(j) b_{i, j}\right) a_{i} c_{i}^{N}(s) \mathrm{d} s \leqslant \gamma_{T} M_{U}^{N}(0) .
$$

But, thanks to $(2.5)$ we have

$$
\sum_{i=2}^{N}\left(U(i)-\sum_{j=1}^{i-1} U(j) b_{i, j}\right) a_{i} c_{i}^{N}=\sum_{i=1}^{N-1} i \sum_{j=i+1}^{N}\left(\frac{U(j)}{j}-\frac{U(i)}{i}\right) a_{j} b_{j, i} c_{j}^{N},
$$

and (3.10) follows at once from (3.13).

Remark 3.4. Notice that the convexity of $U$ entails that $r \mapsto U(r) / r$ is a nondecreasing function. Consequently, each term of the double sum in (3.10) is non-negative.

Lemma 3.5. Let $T \in(0,+\infty)$ and $i \geqslant 1$. There is a constant $\gamma_{i}(T)$ depending only on $K,\left\|c^{0}\right\|_{X}, i$ and $T$ such that, for each $N \geqslant i$,

$$
\left|\frac{\mathrm{d} c_{i}^{N}}{\mathrm{~d} t}\right|_{L^{1}(0, T)} \leqslant \gamma_{i}(T) .
$$


Proof. By (3.1) we have

$$
\begin{aligned}
0 & \leqslant \int_{0}^{T} \sum_{j=1}^{N-i} a_{i+j} b_{i+j, i} c_{i+j}^{N}(s) \mathrm{d} s \\
& \leqslant \int_{0}^{T} a_{i} c_{i}^{N}(s) \mathrm{d} s+\int_{0}^{T} \sum_{j=1}^{N-i} \phi_{i, j} c_{i}^{N}(s) c_{j}^{N}(s) \mathrm{d} s+c_{i}^{N}(T) .
\end{aligned}
$$

From (2.3) and (3.3) we may estimate the right-hand side of the above inequality and obtain

$$
\left|\sum_{j=1}^{N-i} a_{i+j} b_{i+j, i} c_{i+j}^{N}\right|_{L^{1}(0, T)} \leqslant a_{i} T\left\|c^{0}\right\|_{X}+2 K i T\left\|c^{0}\right\|_{X}^{2}+\left\|c^{0}\right\|_{X} .
$$

Estimate (3.14) then follows from (3.1), (2.3), (3.3) and the above estimate.

\section{Existence}

We are now in a position to prove the existence results (Theorems 2.3 and 2.5). To do this we first recall a refined version of the de la Vallée-Poussin theorem for integrable functions [14, Proposition I.1.1].

Theorem 4.1. Let $(\Omega, \mathcal{B}, \mu)$ be a measured space and consider a function $w \in$ $L^{1}(\Omega, \mathcal{B}, \mu)$. Then there exists a function $V \in \mathcal{K}_{1, \infty}$ such that

$$
V(|w|) \in L^{1}(\Omega, \mathcal{B}, \mu)
$$

Remark 4.2. Theorem 4.1 is a classical result when $\mu(\Omega)<\infty$ (see, for example, [5, p. 38]), except for the possibility of choosing $V^{\prime}$ concave. This last fact has been noted in $[\mathbf{1 4}]$.

Proof of Theorem 2.3. We apply Theorem 4.1, $\Omega$ being the set $\mathbb{N} \backslash\{0\}$ and $\mathcal{B}$ the set of all subsets of $\mathbb{N} \backslash\{0\}$. Defining the measure $\mu$ by

$$
\mu(I)=\sum_{i \in I} c_{i}^{0}, \quad I \subset \mathbb{N} \backslash\{0\}
$$

the condition $c^{0} \in X^{+}$ensures that $x \mapsto x$ belongs to $L^{1}(\Omega, \mathcal{B}, \mu)$. By Theorem 4.1 there is thus a function $U_{0} \in \mathcal{K}_{1, \infty}$ such that $x \mapsto U_{0}(x)$ belongs to $L^{1}(\Omega, \mathcal{B}, \mu)$, that is,

$$
\mathcal{U}_{0}:=\sum_{i=1}^{\infty} U_{0}(i) c_{i}^{0}<\infty
$$

In the following we denote by $C$ any positive constant depending only on $K,\left\|c^{0}\right\|_{X}$, $U_{0}$ and $\mathcal{U}_{0}$. The dependence of $C$ upon additional parameters will be indicated explicitly.

By (3.3) and (3.14) the sequence $\left(c_{i}^{N}\right)_{N \geqslant i}$ is bounded in $L^{\infty}(0, T) \cap W^{1,1}(0, T)$ for each $i \geqslant 1$ and $T \in(0,+\infty)$. We then infer from the Helly theorem [10, pp. 372-374] that 
there are a subsequence of $\left(c_{i}^{N}\right)_{N \geqslant i}$, still denoted $\left(c_{i}^{N}\right)_{N \geqslant i}$, and a sequence $c=\left(c_{i}\right)_{i \geqslant 1}$ of functions of locally bounded variation such that

$$
\lim _{N \rightarrow+\infty} c_{i}^{N}(t)=c_{i}(t)
$$

for each $i \geqslant 1$ and $t \geqslant 0$. Clearly, $c_{i}(t) \geqslant 0$ for $i \geqslant 1$ and $t \geqslant 0$ and it follows from (4.2) and (3.3) that $c(t) \in X^{+}$with

$$
\|c(t)\|_{X} \leqslant\left\|c^{0}\right\|_{X}, \quad t \geqslant 0
$$

Furthermore, as $U_{0} \in \mathcal{K}_{1, \infty}$, we infer from (4.1) and Proposition 3.3 that, for each $T \geqslant 0$ and $N \geqslant 3$, there holds

$$
\begin{gathered}
\sum_{i=1}^{N} U_{0}(i) c_{i}^{N}(t) \leqslant C(T), \quad t \in[0, T] \\
0 \leqslant \int_{0}^{T} \sum_{i=1}^{N-1} i \sum_{j=i+1}^{N}\left(\frac{U_{0}(j)}{j}-\frac{U_{0}(i)}{i}\right) a_{j} b_{j, i} c_{j}^{N}(s) \mathrm{d} s \leqslant C(T) .
\end{gathered}
$$

Consider now $T \in(0,+\infty)$ and $M \geqslant 2$. By (4.4), (4.5) and Remark 3.4 we have, for $N \geqslant M+1$,

$$
\begin{gathered}
\sum_{i=1}^{M} U_{0}(i) c_{i}^{N}(t) \leqslant C(T), \quad t \in[0, T], \\
0 \leqslant \int_{0}^{T} \sum_{i=1}^{M-1} i \sum_{j=i+1}^{M}\left(\frac{U_{0}(j)}{j}-\frac{U_{0}(i)}{i}\right) a_{j} b_{j, i} c_{j}^{N}(s) \mathrm{d} s \leqslant C(T) .
\end{gathered}
$$

Due to (4.2) we may pass to the limit as $N \rightarrow+\infty$ in the above estimates and conclude that they both hold true with $c_{i}^{N}$ replaced by $c_{i}$. We next let $M \rightarrow+\infty$ and obtain

$$
\begin{gathered}
\sum_{i=1}^{\infty} U_{0}(i) c_{i}(t) \leqslant C(T), \quad t \in[0, T] \\
0 \leqslant \int_{0}^{T} \sum_{i=1}^{\infty} i \sum_{j=i+1}^{\infty}\left(\frac{U_{0}(j)}{j}-\frac{U_{0}(i)}{i}\right) a_{j} b_{j, i} c_{j}(s) \mathrm{d} s \leqslant C(T) .
\end{gathered}
$$

As a consequence of $(4.3),(2.3),(4.7)$ and the properties of $U_{0}$ we obtain that, for each $i \geqslant 1$,

$$
\sum_{j=1}^{\infty} \phi_{i, j} c_{j} \in L^{1}(0, T) \quad \text { and } \quad \sum_{j=i+1}^{\infty} a_{j} b_{j, i} c_{j} \in L^{1}(0, T) .
$$

We now claim that, for each $i \geqslant 1$, there holds

$$
\begin{aligned}
\lim _{N \rightarrow+\infty}\left|\sum_{j=1}^{N-i} \phi_{i, j} c_{i}^{N} c_{j}^{N}-\sum_{j=1}^{\infty} \phi_{i, j} c_{i} c_{j}\right|_{L^{1}(0, T)} & =0, \\
\lim _{N \rightarrow+\infty}\left|\sum_{j=i+1}^{N} a_{j} b_{j, i} c_{j}^{N}-\sum_{j=i+1}^{\infty} a_{j} b_{j, i} c_{i}\right|_{L^{1}(0, T)} & =0 .
\end{aligned}
$$


Indeed, fix $i \geqslant 1$ and consider $M \geqslant 2$. On the one hand it follows from (4.2), (3.3), (4.3) and the Lebesgue-dominated convergence theorem that

$$
\lim _{N \rightarrow+\infty}\left|\sum_{j=1}^{M} \phi_{i, j}\left(c_{i}^{N} c_{j}^{N}-c_{i} c_{j}\right)\right|_{L^{1}(0, T)}=0 .
$$

On the other hand we infer from (3.3), (2.3) and (4.4) that, for $N \geqslant i+M+1$,

$$
\begin{aligned}
&\left|\sum_{j=M+1}^{N-i} \phi_{i, j} c_{i}^{N} c_{j}^{N}\right|_{L^{1}(0, T)} \leqslant K i\left\|c^{0}\right\|_{X}\left|\sum_{j=M+1}^{N-i} j c_{j}^{N}\right|_{L^{1}(0, T)} \\
& \leqslant C(i, T) \sup _{j \geqslant M} \frac{j}{U_{0}(j)}\left|\sum_{j=M+1}^{N-i} U_{0}(j) c_{j}^{N}\right|_{L^{1}(0, T)}, \\
&\left|\sum_{j=M+1}^{N-i} \phi_{i, j} c_{i}^{N} c_{j}^{N}\right|_{L^{1}(0, T)} \leqslant C(i, T) \sup _{j \geqslant M} \frac{j}{U_{0}(j)} .
\end{aligned}
$$

Similarly, (4.3), (2.3) and (4.6) yield

$$
\left|\sum_{j=M+1}^{\infty} \phi_{i, j} c_{i} c_{j}\right|_{L^{1}(0, T)} \leqslant C(i, T) \sup _{j \geqslant M} \frac{j}{U_{0}(j)} .
$$

Combining (4.11)-(4.13) we obtain

$$
\limsup _{N \rightarrow+\infty}\left|\sum_{j=1}^{N-i} \phi_{i, j} c_{i}^{N} c_{j}^{N}-\sum_{j=1}^{\infty} \phi_{i, j} c_{i} c_{j}\right|_{L^{1}(0, T)} \leqslant C(i, T) \sup _{j \geqslant M} \frac{j}{U_{0}(j)}
$$

for every $M \geqslant 2$. Recalling that $U_{0}$ belongs to $\mathcal{K}_{1, \infty}$, we see that the right-hand side of the above inequality converges to zero as $M \rightarrow+\infty$, hence (4.9).

We next turn to the proof of (4.10). Consider $i \geqslant 1$ and $\varepsilon \in(0,1)$. As $U_{0} \in \mathcal{K}_{1, \infty}$ there is $M \geqslant i$ such that

$$
j \geqslant M \Longrightarrow\left(\frac{U_{0}(j)}{j}-\frac{U_{0}(i)}{i}\right) \geqslant \frac{1}{\varepsilon} .
$$

For $N \geqslant M$ we infer from (4.5), (4.7) and (4.14) that

$$
\begin{gathered}
\int_{0}^{T} \sum_{j=M+1}^{N} a_{j} b_{j, i} c_{j}^{N}(s) \mathrm{d} s \leqslant \varepsilon C(T), \\
\int_{0}^{T} \sum_{j=M+1}^{\infty} a_{j} b_{j, i} c_{j}(s) \mathrm{d} s \leqslant \varepsilon C(T) .
\end{gathered}
$$

It also follows from (4.2), (3.3), (4.3) and the Lebesgue-dominated convergence theorem that

$$
\lim _{N \rightarrow+\infty}\left|\sum_{j=i+1}^{M} a_{j} b_{j, i} c_{j}^{N}-\sum_{j=i+1}^{M} a_{j} b_{j, i} c_{j}\right|_{L^{1}(0, T)}=0 .
$$


Consequently, by (4.15)-(4.17) we have

$$
\limsup _{N \rightarrow+\infty}\left|\sum_{j=i+1}^{N} a_{j} b_{j, i} c_{j}^{N}-\sum_{j=i+1}^{\infty} a_{j} b_{j, i} c_{j}\right|_{L^{1}(0, T)} \leqslant \varepsilon
$$

hence (4.10) after letting $\varepsilon \rightarrow 0$.

Due to (4.2), (3.3), (4.3), (4.9) and (4.10) it is now straightforward to check that $c_{i}$ satisfies Definition 2.2 (ii) for each $i \geqslant 1$. Recalling (4.8), the continuity of $c_{i}$ then follows and we have thus shown that $c=\left(c_{i}\right)$ is a solution to $(2.1),(2.2)$ on $[0,+\infty)$. In order to complete the proof of Theorem 2.3 it remains to check (2.6). Let $t \in(0,+\infty)$. For $N \geqslant M \geqslant 3$ we have by (3.3) that

$$
\left|\|c(t)\|_{X}-\left\|c^{0}\right\|_{X}\right| \leqslant \sum_{i=1}^{M} i\left|c_{i}^{N}(t)-c_{i}(t)\right|+\sum_{i=N+1}^{\infty} i c_{i}^{0}+\sum_{i=M+1}^{N} i c_{i}^{N}(t)+\sum_{i=M+1}^{\infty} i c_{i}(t) .
$$

It then follows from (4.4) and (4.6) that

$$
\left|\|c(t)\|_{X}-\left\|c^{0}\right\|_{X}\right| \leqslant \sum_{i=1}^{M} i\left|c_{i}^{N}(t)-c_{i}(t)\right|+\sum_{i=N+1}^{\infty} i c_{i}^{0}+C(T) \sup _{i \geqslant M} \frac{i}{U_{0}(i)} .
$$

Since $c^{0} \in X^{+}$, we first deduce from (4.2) that

$$
\left|\|c(t)\|_{X}-\left\|c^{0}\right\|_{X}\right| \leqslant C(T) \sup _{i \geqslant M} \frac{i}{U_{0}(i)} .
$$

Recalling that $U_{0} \in \mathcal{K}_{1, \infty}$, we conclude that $\|c(t)\|_{X}=\left\|c^{0}\right\|_{X}$, and the proof of Theorem 2.3 is complete.

Proof of Theorem 2.5. We only need to show that the solution we constructed in the proof of Theorem 2.3 enjoys the additional property (2.11). But, as $U \in \mathcal{K}_{1} \cup \mathcal{K}_{2}$, (2.11) follows at once from Proposition 3.3 and (4.2).

\section{Uniqueness}

We end this paper with a partial uniqueness result inspired by [1, Theorem 4.2]. A different approach to uniqueness may be found in $[\mathbf{2 0}]$ in the pure coagulation case $\left(a_{i} \equiv 0\right)$.

Proposition 5.1. Consider $c^{0} \in X^{+}, T \in(0,+\infty)$ and assume that the rate coefficients $\left(\phi_{i, j}\right),\left(a_{i}\right)$ and $\left(b_{i, j}\right)$ satisfy the assumptions (2.3)-(2.5). Assume further that there are a sequence $\left(\varphi_{i}\right)_{i \geqslant 1}$ of non-negative real numbers and $\kappa>0$ such that

$$
\varphi_{i} \leqslant \kappa i \quad \text { and } \quad \phi_{i, j} \leqslant \varphi_{i}+\varphi_{j}, \quad i, j \geqslant 1 .
$$

Then there is at most one solution $c$ to $(2.1),(2.2)$ on $[0, T)$ such that

$$
\|c(t)\|_{X}=\left\|c^{0}\right\|_{X} \quad \text { and } \quad \sum_{i=1}^{\infty} i \varphi_{i} c_{i} \in L^{1}(0, t) \quad \text { for each } t \in(0, T) .
$$


Combining Proposition 5.1 and Theorem 2.5 we obtain the following result.

Corollary 5.2. Consider $c^{0} \in X^{+}$and assume that the rate coefficients $\left(\phi_{i, j}\right),\left(a_{i}\right)$ and $\left(b_{i, j}\right)$ satisfy the assumptions (2.3)-(2.5). Assume further that there are $\alpha \in[0,1]$ and $K_{\alpha}>0$ such that

$$
\sum_{i=1}^{\infty} i^{1+\alpha} c_{i}^{0}<\infty \quad \text { and } \quad \phi_{i, j} \leqslant K_{\alpha}\left(i^{\alpha}+j^{\alpha}\right), \quad i, j \geqslant 1 .
$$

Then there is one and only one solution $c$ to (2.1), (2.2) on $[0,+\infty)$ satisfying (2.6) and, for each $T \in(0,+\infty)$,

$$
\sup _{t \in[0, T]} \sum_{i=1}^{\infty} i^{1+\alpha} c_{i}(t)<\infty
$$

For the discrete coagulation-fragmentation equations (1.1), (1.2), the above result has been obtained in [1, Theorem 4.2] for $\alpha=0$.

Proof. As $r \mapsto r^{1+\alpha}$ belongs to $\mathcal{K}_{1}$, the existence of a solution to (2.1), (2.2) on $[0,+\infty)$ satisfying (2.6) and (5.4) follows from Theorem 2.5. The uniqueness of such a solution is then a consequence of Proposition 5.1 with $\varphi_{i}=K_{\alpha} i^{\alpha}$ after noting that (5.4) entails (5.2).

The first step towards the proof of Proposition 5.1 is the following lemma.

Lemma 5.3. Under the assumptions of Proposition 5.1 any solution $c$ to (2.1), (2.2) on $[0, T)$ satisfying (5.2) also satisfies, for $t \in(0, T)$,

$$
\begin{aligned}
\lim _{N \rightarrow+\infty} \int_{0}^{t} \sum_{i=N+1}^{\infty}\left(\sum_{j=1}^{N} j b_{i, j}\right) a_{i} c_{i}(s) \mathrm{d} s & =0, \\
\lim _{N \rightarrow+\infty} \int_{0}^{t} \sum_{i=1}^{N} \sum_{j=N+1-i}^{\infty} i \phi_{i, j} c_{i}(s) c_{j}(s) \mathrm{d} s & =0 .
\end{aligned}
$$

Proof. Fix $t \in(0, T)$. By (5.1) we have

$$
i \phi_{i, j} c_{i}(s) c_{j}(s) \leqslant i \varphi_{i} c_{i}(s) c_{j}(s)+i c_{i}(s) \varphi_{j} c_{j}(s)
$$

for $s \in(0, t)$, and we easily deduce from (5.2) that (5.6) holds true.

Proceeding next as in [1, Lemma 3.1], we infer from (2.1) that, for $N \geqslant 2$,

$$
\sum_{i=1}^{N} i c_{i}(t)=\sum_{i=1}^{N} i c_{i}^{0}+\int_{0}^{t} \sum_{i=N+1}^{\infty}\left(\sum_{j=1}^{N} j b_{i, j}\right) a_{i} c_{i}(s) \mathrm{d} s-\int_{0}^{t} \sum_{i=1}^{N} \sum_{j=N+1-i}^{\infty} i \phi_{i, j} c_{i}(s) c_{j}(s) \mathrm{d} s .
$$

As $c$ is a density-conserving solution by (5.2), we may let $N \rightarrow+\infty$ in the above identity to obtain

$$
\lim _{N \rightarrow+\infty} \int_{0}^{t}\left(\sum_{i=N+1}^{\infty}\left(\sum_{j=1}^{N} j b_{i, j}\right) a_{i} c_{i}(s)-\sum_{i=1}^{N} \sum_{j=N+1-i}^{\infty} i \phi_{i, j} c_{i}(s) c_{j}(s)\right) \mathrm{d} s=0 .
$$

Combining (5.6) and the above identity yield (5.5). 
Proof of Proposition 5.1. We only sketch the proof below as it follows the lines of the proof of [1, Theorem 4.2]. Consider two solutions $c=\left(c_{i}\right)_{i \geqslant 1}$ and $\hat{c}=\left(\hat{c}_{i}\right)_{i \geqslant 1}$ to (2.1), $(2.2)$ on $[0, T)$ satisfying (5.2). For $i \geqslant 1$ we put

$$
z_{i}=c_{i}-\hat{c}_{i} \quad \text { and } \quad g_{i}=i \operatorname{sign}\left(z_{i}\right),
$$

where $\operatorname{sign}(r)=r /|r|$ if $r \in \mathbb{R} \backslash\{0\}$ and $\operatorname{sign}(0)=0$. Fix $N \geqslant 2$ and $t \in(0, T)$. Proceeding as in $[\mathbf{1}$, Theorem 4.2$]$ we infer from (2.1) that

$$
\begin{aligned}
\sum_{i=1}^{N} i\left|z_{i}(t)\right|=\frac{1}{2} & \int_{0}^{t} \sum_{i=1}^{N-1} \sum_{j=1}^{N-i}\left(g_{i+j}-g_{i}-g_{j}\right) \phi_{i, j}\left(c_{i} z_{j}+\hat{c}_{j} z_{i}\right) \mathrm{d} s \\
& -\int_{0}^{t} \sum_{i=2}^{N}\left(g_{i}-\sum_{j=1}^{i-1} g_{j} b_{i, j}\right) a_{i} z_{i} \mathrm{~d} s-\int_{0}^{t} \sum_{i=1}^{N} \sum_{j=N+1-i}^{\infty} g_{i} \phi_{i, j}\left(c_{i} c_{j}-\hat{c}_{i} \hat{c}_{j}\right) \mathrm{d} s \\
& +\int_{0}^{t} \sum_{i=N+1}^{\infty}\left(\sum_{j=1}^{N} g_{j} b_{i, j}\right) a_{i} z_{i} \mathrm{~d} s .
\end{aligned}
$$

Since $|\operatorname{sign}(r)| \leqslant 1$ for any $r \in \mathbb{R}$ we have

$$
\begin{aligned}
& \left(g_{i+j}-g_{i}-g_{j}\right) z_{j}=\left((i+j) \operatorname{sign}\left(z_{i+j} z_{j}\right)-i \operatorname{sign}\left(z_{i} z_{j}\right)-j\right)\left|z_{j}\right|, \\
& \left(g_{i+j}-g_{i}-g_{j}\right) z_{j} \leqslant 2 i\left|z_{j}\right| .
\end{aligned}
$$

We next deduce from (2.5) that

$$
\left(g_{i}-\sum_{j=1}^{i-1} g_{j} b_{i, j}\right) z_{i}=\left(i-\sum_{j=1}^{i-1} j \operatorname{sign}\left(z_{i} z_{j}\right) b_{i, j}\right)\left|z_{i}\right| \geqslant 0 .
$$

We now infer from (5.1), (5.7) and (5.8) that

$$
\begin{aligned}
\sum_{i=1}^{N} i\left|z_{i}(t)\right| \leqslant(1+\kappa) \int_{0}^{t}( & \left.\sum_{i=1}^{N} i \varphi_{i}\left(c_{i}+\hat{c}_{i}\right)\right)\left(\sum_{j=1}^{N} j\left|z_{j}\right|\right) \mathrm{d} s \\
& +\int_{0}^{t} \sum_{i=1}^{N} \sum_{j=N+1-i}^{\infty} i \phi_{i, j}\left(c_{i} c_{j}+\hat{c}_{i} \hat{c}_{j}\right) \mathrm{d} s \\
& +\int_{0}^{t} \sum_{i=N+1}^{\infty}\left(\sum_{j=1}^{N} j b_{i, j}\right) a_{i}\left(c_{i}+\hat{c}_{i}\right) \mathrm{d} s
\end{aligned}
$$

According to (5.2) and Lemma 5.3 we may pass to the limit as $N \rightarrow+\infty$ in the above inequality and obtain

$$
\sum_{i=1}^{\infty} i\left|z_{i}(t)\right| \leqslant(1+\kappa) \int_{0}^{t}\left(\sum_{i=1}^{\infty} i \varphi_{i}\left(c_{i}+\hat{c}_{i}\right)\right)\left(\sum_{j=1}^{\infty} j\left|z_{j}\right|\right) \mathrm{d} s .
$$


Thanks to (5.2) we may use the Gronwall lemma to conclude that

$$
\sum_{i=1}^{\infty} i\left|z_{i}(t)\right|=0, \quad t \in[0, T),
$$

hence $c \equiv \hat{c}$.

\section{References}

1. J. M. BALL AND J. CARR, The discrete coagulation-fragmentation equations: existence, uniqueness, and density conservation, J. Stat. Phys. 61 (1990), 203-234.

2. J. CARR, Asymptotic behaviour of solutions to the coagulation-fragmentation equations, I, The strong fragmentation case, Proc. R. Soc. Edinb. A 121 (1992), 231-244.

3. J. CARR AND F. P. DA Costa, Asymptotic behavior of solutions to the coagulationfragmentation equations, II, Weak fragmentation, J. Stat. Phys. 77 (1994), 89-123.

4. F. P. DA Costa, Existence and uniqueness of density conserving solutions to the coagulation-fragmentation equations with strong fragmentation, J. Math. Analysis Appl. 192 (1995), 892-914.

5. C. Dellacherie and P. A. Meyer, Probabilités et potentiel, Chapitres 1-4 (Hermann, Paris, 1975).

6. R. L. Drake, A general mathematical survey of the coagulation equation, in Topics in current aerosol research, part 2, International Reviews in Aerosol Physics and Chemistry, pp. 203-376 (Pergamon Press, Oxford, 1972).

7. O. J. Heilmann, Analytical solutions of Smoluchowski's coagulation equation, J. Phys. A 25 (1992), 3763-3771.

8. E. M. HendRiks, M. H. ERnst And R. M. ZifF, Coagulation equations with gelation, J. Stat. Phys. 31 (1983), 519-563.

9. I. JEON, Existence of gelling solutions for coagulation-fragmentation equations, Commun. Math. Phys. 194 (1998), 541-567.

10. A. N. Kolmogorov AND S. V. Fomin, Introductory real analysis (Prentice-Hall, Englewood Cliffs, NJ, 1970).

11. M. A. Krasnosel'skit and Ya. B. Rutickit, Convex functions and Orlicz spaces (Noordhoff, Groningen, 1961).

12. Ph. Laurençot, Global solutions to the discrete coagulation equations, Mathematika 46 (1999), in press.

13. Ph. Laurençot, The Lifshitz-Slyozov equation with encounters, Math. Models Methods Appl. Sci. 11 (2001), in press.

14. LÊ CHÂU-Hò̀n, Etude de la classe des opérateurs m-accrétifs de $L^{1}(\Omega)$ et accrétifs dans $L^{\infty}(\Omega)$, Thèse de troisième cycle, Université de Paris VI, 1977.

15. F. Leyvraz and H. R. Tschudi, Singularities in the kinetics of coagulation processes, J. Phys. A 14 (1981), 3389-3405.

16. F. Leyvraz and H. R. Tschudi, Critical kinetics near gelation, J. Phys. A 15 (1982), 1951-1964.

17. E. D. McGrady And R. M. Ziff, 'Shattering' transition in fragmentation, Phys. Rev. Lett. 58 (1987), 892-895.

18. J. B. MCLEOD, On an infinite set of non-linear differential equations, Q. J. Math. Oxford (2) 13 (1962), 119-128.

19. J. B. MCLeOD, On an infinite set of non-linear differential equations, II, Q. J. Math. Oxford (2) 13 (1962), 193-205.

20. J. R. NORRIS, Smoluchowski's coagulation equation: uniqueness, non-uniqueness and a hydrodynamic limit for the stochastic coalescent, Ann. Appl. Prob. 9 (1999), 78-109. 
21. M. Smoluchowski, Drei Vorträge über Diffusion, Brownsche Molekularbewegung und Koagulation von Kolloidteilchen, Physik. Z. 17 (1916), 557-599.

22. M. Smoluchowski, Versuch einer mathematischen Theorie der Koagulationskinetik kolloider Lösungen, Z. Phys. Chem. 92 (1917), 129-168.

23. J. L. Spouge, An existence theorem for the discrete coagulation-fragmentation equations, Math. Proc. Camb. Phil. Soc. 96 (1984), 351-357.

24. W. H. White, A global existence theorem for Smoluchowski's coagulation equations, Proc. Am. Math. Soc. 80 (1980), 273-276. 